\title{
Comparison of the Psychological Status of Chronic Pain Patients and the General Population
}

\author{
Laxmaiah Manchikanti, MD*, Bert Fellows, MA\#, Vidyasagar Pampati, MSc ${ }^{\star *}$, Carla Beyer, RN"\#, Kim \\ Damron, RN"\#, and Renee C. Barnhill, RN"\#
}

This study was designed to evaluate the psychological status of 50 individuals without chronic pain and without psychotherapeutic drug therapy, Group I, the control group; and Group II, a chronic pain group with 100 chronic pain patients. All the participants were tested utilizing Millon Clinical Multiaxial Inventory III (MCMI-III). Results were analyzed and compared for various clinical personality patterns, including personality traits and personality disorders; severe personality pathology for schizotypal, borderline and paranoid personality pathology; and multiple clinical syndromes, including generalized anxiety disorder, somatization disorder, major depression, bipolar manic disorder and dysthymic disorder, etc.

There were no significant differences noted in clinical personality patterns or severe personality pathology. In the analysis of clinical syndromes, generalized anxiety disor- der ( $40 \%$ vs $14 \%$ ), somatization disorder (26\% vs $0 \%$ ), and major depression ( $22 \%$ vs $4 \%$ ) were seen in a greater proportion of patients in the chronic pain group. The prevalence of psychological disorders in the control group was $24 \%$, compared to $55 \%$ in chronic pain group.

In conclusion, this evaluation showed that clinical personality patterns are present in both groups of patients. Psychological abnormalities such as generalized anxiety disorder, somatization disorder, and major depression are commonly seen in chronic pain patients.

Keywords: Chronic pain, psychological evaluation, depression, generalized anxiety disorder, somatization disorder, personality disorders, Millon Clinical Multiaxial Inventory-III

ance of a psychosocial basis. Fishbain et al (28), in a metaanalysis of previous studies of chronic pain and depression, examined a total of 191 studies and reviewed in detail 83 studies, concluding that 21 of 23 articles related the severity of pain to the degree of depression. In addition, the duration of pain was also related to the development of depression in three of the three articles that included patients with multiple types of symptomatology. Thus, depression is a hallmark of chronic pain. However, biomedical purists or organic reductionists maintain that depression is a rational consequence of unrelenting, undiagnosed pain. In contrast, psychosocial purists or psychological reductionists maintain that the depression is a feature of personality prone to chronic pain and its result in disability. Fishbain et al (28) presented strong evidence indicating that depression is a feature secondary to chronic pain. They also showed greater support for the consequence and scar hypothesis than for the antecedent hypothesis in regard to the relationship between pain and depression. Even though many psychiatric diagnoses have been described in association with chronic pain, depressive disorders and anxiety disorders are predominant. The evidence on diagnosis of somatoform disorders, particularly somatization 
disorder, is conflicting $(3,4,7,29-31)$.

A considerable amount of research has been devoted to profiling the psychological and behavioral characteristics of chronic pain patients using numerous psychological instruments (32). These include, but are not limited to, measures of personality, mood, less ability in coping, and pain impact. Ideally, patient evaluation of psychological profiles should be useful for classifying individual patients, determining treatment strategies, and predicting treatment response, apart from developing a better understanding of the psychological mechanisms mediating the chronic pain experience.

The clinical results of interventional procedures are often mediated by several factors in addition to physical illness, including the patient's emotional status, social environment, lifestyle, and incentive for improvement. Many of these factors have been shown to be predictive of poor surgical outcome (33). However, Carragee (34) showed that psychological screening seems to be most useful in those patients with lesser degrees of disc pathologic findings, longer disability, and confounding economic issues.

Assessment of psychological status is achieved using interviews and self-report instruments. Ideally, psychological evaluation is performed by a trained psychologist or a psychiatrist with interview and utilization of self-report instruments. This requires identification of the patients requiring the services of a psychologist or a psychiatrist and availability of psychiatrists and psychologists interested in pain management in the community, and final feasibility rests on financial resources. The comprehensive psychological interview and evaluation are extensive and expensive, not covered by many insurers, resulting in practical problems for interventional pain physicians. Clinical trials have established the efficacy of antidepressant medications and specific psychotherapies for depressive psychiatric and primary care patients when care is provided by trained research personnel or under standarized protocols (35-40).

Comprehensive psychological evaluation is performed only by mental health professionals including psychologists and psychiatrists. Similarly, the Minnesota Multiphasic Personality Inventory (MMPI) also should be administered under the supervision of a clinical psychologist. Further, the MMPI-II profile of a patient in pain has significantly different meaning than the identical test profile of a person who presents with primary symptoms other than pain. Thus, MMPI-II administration requires experience to properly use the test, with specific and advanced training in pain psychology. In addition, various other tests have also been utilized with increasing frequency in interventional pain management. There are multiple instruments which can be used by physicians, nurses, and other qualified clinicians, along with psychologists and psychiatrists.

The Millon Clinical Multiaxial Inventory (MCMI) is an evolving assessment tool. Numerous features are distinguished in the MCMI-III from other inventories, including its relative brevity, its theoretical anchoring, its structural characteristics, and the use of a three-stage validation framework. The MCMI-III may be used by psychologists and other qualified mental health professionals in clinical or counseling settings where individuals are being evaluated for emotional, behavioral or interpersonal difficulties. It can be used to assess clinical and personality disorders and to guide treatment decisions by providing an integrated picture of personality characteristics and clinical syndromes. The MCMI-III Manual states that individuals who use the MCMI and its associated reports or supervise its use should have sufficient background in tectologic and psychometric methods, and clinical practice and theory to understand test manuals. However, the MCMI-III Manual also states that uses of the Millon computer-based reports register high levels of satisfaction with their overall quality and with their correspondence to independently derive with clinical observations and judgments. It is cautioned, nevertheless, that clinicians who use the interpretative report should not be lulled over time into uncritical acceptance; they should routinely compare the statements generated against independent clinical evidence (41). However, the findings of recent investigations into the validity of MCMI interpretative reports provide strong evidence that ratings of its accuracy are higher than can be accounted for by the Barnum effect or the computer-generated format (41-44). Considering the fact that many chronic pain patients suffer with mental disorders, their comprehensive evaluation is not feasible; and psychological evaluation has not been conducted in patients presenting in an interventional pain medicine setting and compared to a nonpatient population, specifically utilizing a screening evaluation. Hence, this screening evaluation was undertaken to evaluate the psychological status of chronic pain patients involved in an interventional pain medicine setting and to compare them with individuals without history of chronic pain or a psychotherapeutic drug therapy, present or past, utilizing a standarized major psychological instrument the MCMI-III. The purpose of the study was to see if patients presenting to an interventional pain medicine setting are different from normal individuals without pain 
Table 1: Clinical personality patterns

\begin{tabular}{lcccc}
\hline & \multicolumn{2}{c}{ Personality } & Traits & \multicolumn{2}{c}{ Personality Disorders } \\
& Group I & Group II & Group I & Group II \\
\hline Compulsive & $22 \%$ & $12 \%$ & $16 \%$ & $10 \%$ \\
Histrionic & $10 \%$ & $12 \%$ & $24 \%$ & $9 \%$ \\
Narcissistic & $10 \%$ & $5 \%$ & $4 \%$ & $4 \%$ \\
Dependent & $2 \%$ & $11 \%$ & $12 \%$ & $10 \%$ \\
Avoidant & $2 \%$ & $7 \%$ & $2 \%$ & $10 \%$ \\
Schizoid & $0 \%$ & $2 \%$ & $2 \%$ & $9 \%$ \\
Negativistic & $2 \%$ & $9 \%$ & $0 \%$ & $3 \%$ \\
Sadistic & $2 \%$ & $2 \%$ & $0 \%$ & $1 \%$ \\
Antisocial & $2 \%$ & $2 \%$ & $0 \%$ & $2 \%$ \\
Masochistic & $0 \%$ & $1 \%$ & $0 \%$ & $0 \%$ \\
At least One abnormality & $62 \%$ & $62 \%$ & $42 \%$ & $50 \%$ \\
\hline Group I- Control Group II - Chronic Pain & & & &
\end{tabular}

and also to evaluate the feasibility of the MCMI-III as a screening test for patients undergoing interventional pain procedures.

\section{METHODS}

This study was designed to evaluate the psychological status of 50 individuals without chronic pain and psychotherapeutic drug therapy (Group I), the control group; and Group II, or a group of 100 chronic pain patients presenting to one private interventional pain management practice in a nonuniversity setting. The control group was recruited from a nonpain patient population of employees, their spouses, and relatives, by posting a notice at the center. Patients younger than 18 years or older than 90 years, those who had had pain for less than 6 months, and those who were unable to undergo psychological evaluation and testing were excluded from the pain patient group. In the control group, if the patients suffered with any type of pain, chronic or acute, were on any psychotherapeutic drugs, and had had any history of major psychological problems in the past, or received any type of psychological management, they were excluded. There was no remuneration for any of the participants.

Patients in both groups, Group I, the control group, with no pain and Group II, the chronic pain group, were evaluated with the MCMI-III. It was given to all participants in both groups, along with an explanation of the nature of the test and utilization of the data for purposes of publication. Patients in Group II also understood that the results would be utilized in their management. One hundred consecutive patients who met inclusion criteria and agreed to participate were included in Group II.

Following completion of the study, data were analyzed for various aspects by a statistician without knowledge of who the participants were. Data were recorded on a database using Microsoft Access ${ }^{\circledR}$, the SPSS version 9.0 statistical package. This package was used to generate the frequency tables, and the chi-squared statistic was used to test the significance difference between groups, Fischer's exact test was used wherever expected value was less than five. Results were considered statistically significant if the $p$ value was less than 0.05 .

\section{RESULTS}

There were no differences noted in the participant characteristics of age or gender.

\section{Personality Patterns}

As shown in Table 1, personality patterns (traits, and disorders) were analyzed for schizoid, avoidant, dependent, histrionic, narcissistic, antisocial, sadistic, compulsive, negativistic, and masochistic characteristics. No significant differences were identified among groups. 
Table 2. Severe personality pathology

\begin{tabular}{lcc}
\hline & \multicolumn{2}{c}{ Personality Pathology } \\
& Group I & Group II \\
\hline Borderline & $6 \%$ & $16 \%$ \\
Paranoid & $4 \%$ & $11 \%$ \\
Schizotypal & $2 \%$ & $7 \%$ \\
At least one abnormality & $12 \%$ & $31 \%$ \\
\hline Group I - Control Group II - Chronic Pain &
\end{tabular}

Table 2 shows severe personality pathology, without significant differences among groups.

\section{Clinical Syndromes}

The MCMI-III denotes clinical syndromes, as well as severe clinical syndromes. While thought disorder, major depression and delusion disorder are considered as severe clinical syndromes; anxiety disorder, somatoform disorder, bipolar manic disorder, dysthymic disorder, alcohol dependence, drug dependence, and post-traumatic stress were considered as clinical syndromes. However, in the category of severe clinical syndromes, including thought disorder, major depression and delusion disorder, only major depression was of any importance. Hence, the results of clinical syndromes and severe clinical syndromes were combined, with the addition of major depression to clinical syndromes and elimination of thought disorder and delusional disorder, which were present in only $1 \%$ of the patients in Group II.

As shown in Table 3, a significantly greater proportion of patients in Group II presented with generalized anxiety disorder $(40 \%)$, somatization disorder $(26 \%)$, and major depression $(22 \%)$.

\section{DISCUSSION}

Patients with chronic pain compared to patients without pain or psychotherapeutic drug therapy showed no significant differences in terms of clinical personality patterns or severe personality pathology. However, significant differences were noted, with the clinical syndromes consisting of a triad of generalized anxiety disorder, major depression, and somatization disorder. In addition, only $24 \%$ of the normal population presented with a diagnosable mental disorder using the MCMI, in contrast to $55 \%$ of the population suffering with chronic pain. The goal of the study was to describe the association between mental dis-
Table 3. Clinical syndromes

\begin{tabular}{lcc}
\hline & \multicolumn{2}{c}{ Clinical Syndrome } \\
& Group I & Group II \\
\hline Generalized anxiety disorder & $14 \%$ & $40 \% *$ \\
Somatization disorder & $0 \%$ & $26 \% *$ \\
Major depression & $4 \%$ & $22 \% *$ \\
Bipolar: manic disorder & $4 \%$ & $2 \%$ \\
Alcohol dependence & $0 \%$ & $5 \%$ \\
Post-traumatic stress & $2 \%$ & $2 \%$ \\
Dysthymic disorder & $0 \%$ & $2 \%$ \\
At least One abnormality & $14 \%$ & $51 \% *$ \\
\hline Group I-Control Group II - Chronic Pain * Indicates significant difference
\end{tabular}

orders and chronic pain in an interventional pain medicine setting.

Polatin et al (23) in evaluating the relationship of psychopathology and chronic low back pain in 200 patients, showed that depressive disorders accounted for $49 \%$ of current prevalence and $68 \%$ of lifetime prevalence in chronic low back pain patients; whereas anxiety disorders were present in $15 \%$ of the patients, with substance abuse disorders in $19 \%$. Kramlinger et al (45) showed current major depressive rates as $25 \%$, which was definite, and $39 \%$ with a probable evidence. Manchikanti et al (24) in evaluating 200 low back pain patients in an interventional pain management setting, showed that $65 \%$ of the patients presented with one or more psychological abnormalities, excluding personality traits or disorders. They also reported generalized anxiety disorder in $49 \%$ of the patients, depressive disorders in $53 \%$ of the patients, and somatization disorder in $34 \%$ of the patients. Apart from clinical disorders such as depression, generalized anxiety disorder and somatization disorder, the influence of personality on pain experience has long interested clinicians working with individuals having chronic pain (46). Many early theories of chronic pain also maintained that personality played an important role in the development and maintenance of chronic pain conditions (47-51). The early psychological literature on chronic pain focused on the relationship of personality to pain, and significant writings about personality and pain were based on a model of personality that emphasized the influence of personality traits or dispositions that are present not only in chronic pain patients, but also in the population at large (46). 
The present study sought to compare the psychological status of patients suffering with chronic persistent pain with that of individuals without pain. Surprisingly, our results showed that various types of personality patterns and personality disorders were not significantly different in the two groups of patients. Thus, it is difficult to associate personality pattern or pathology with chronic pain. It is possible that the role of personality patterns, disorders or pathology in chronic pain, specifically in the interventional pain management setting, is unclear and, at best, only hypothetical.

The incidence of generalized anxiety disorder in $40 \%$ of patients in the present study is similar to the results of generalized anxiety disorder in our previous evaluation of low back pain patients with $49 \%$ incidence (24). However, Polatin et al (23) showed anxiety disorders to be present only in $15 \%$ of patients. The $14 \%$ incidence of anxiety disorder in the present study in the control group is also similar to the incidence of $13 \%$ in a population in the 18 to 54 age group in the US population (52). Asmundson et al (53) also showed that only $18 \%$ of the patients with chronic musculoskeletal pain were diagnosed with a current anxiety disorder. Atkinson et al (15) however, comparing patients with low back pain to a matched sample of pain-free men, found that the chronic pain groups had significantly higher lifetime prevalence rates of major anxiety disorder of $31 \%$ vs. $14 \%$, which is similar to results in this study.

Depressive disorder was shown to be present in $26 \%$ of the population with chronic pain (major depression $22 \%$, dysthymia $2 \%$ and bipolar manic disorder $2 \%$ ), whereas it was present in only $8 \%$ of individuals without chronic pain (major depression 4\%, dysthymia $0 \%$, and bipolar manic disorder 4\%). Statistics show that approximately $10 \%$ of the US population age 18 and older has a depressive disorder, whereas 5\% suffer with major depression (54-56). The results in pain patients were similar to the previous reports of Fishbain et al (28), with presence of depression in $30 \%$ of the patients; to our previous results in low back pain patients, with an incidence of $23 \%$ (24), and to those of Magni et al (25), with findings of depression in $18 \%$ of patients. However, the results showed a lesser incidence compared to the previous findings of Polatin et al (23), showing the presence of major depressive disorder in $49 \%$ of the population, in patients with low back pain.

Similar to anxiety disorders and depression, somatization disorder appears to be common in chronic pain patients. Somatization disorder is not only a complex disorder, but also a complicated and controversial psychiatric diagno- sis. Aronoff et al (57) questioned the validity of pain disorder in somatization disorder as diagnostic entities. Patients with a tendency towards somatization may present to physicians hoping to obtain medical attention and symptomatic treatment. This tendency essentially begins in childhood and is believed to account for a significant proportion of medical care utilization in adults. Fink et al (58) showed that between $22 \%$ and $58 \%$ of the consecutive patients in primary care fulfill the diagnostic criteria for a somatization disorder. Katon et al (59) diagnosed somatization disorder in $16 \%$ of the patients. In our previous evaluation (24), we showed that somatization disorder was present in $34 \%$ of the patients, with no significant difference between men and women in patients who were suffering with chronic low back pain. Our present results of $26 \%$ incidence of somatization disorder in chronic pain patients is somewhat similar to some studies, whereas it is lower than in other studies.

As a whole, our data does not support correlation between personality features, disorders, pathology and chronic pain. However, it supports the association between chronic pain and multiple psychological disorders, including generalized anxiety disorder, major depression, and somatization disorder. Obviously, in a significant number of patients, there is a physical problem associated with an emotional issue. Thus, we recommend that patients presenting with chronic pain to an interventional pain management setting must undergo psychological evaluation evaluating three dimensions, namely, generalized anxiety disorder, depression and somatization disorder.

Our study may be criticized for not depending on provider evaluation for screening purposes; omitting clinical interview by a psychologist; and, finally, utilizing a single selfreporting instrument. We argue that much of the information obtained in pain management, including the information obtained from comprehensive pain questionnaires, even though exhaustive, is not sufficient to evaluate psychological condition of a chronic pain patient. Further, it is sobering to note that Waddell and Turk (60), while emphasizing the importance of psychological evaluation, asserted that psychological diseases cannot be assessed reliably by general physicians vs surgeons vs clinical impression. Waddell further stated that, in spite of his extensive research experience in the field of low back pain management and diagnosis, when he compared his rating of depression from his general clinical interview with the patient's score on a psychological questionnaire evaluating major depressive symptoms, his clinical judgment was hopelessly "inaccurate" (60). 
We have not utilized a psychological interview in conjunction with MCMI-III evaluation, as our purpose was to evaluate psychological disorders based on a self-reporting instrument for screening purposes. However, further clinician's judgment may selectively refer patients for comprehensive psychological evaluation and subsequent management. Psychologists and psychiatrists may tend to disagree with this approach. However, to maintain appropriate access and manage issues of psychological importance in clinical practice, this approach is essential and also costeffective.

The third criticism may be utilization of a single instrument, namely, the MCMI-III. Multiple psychological evaluations have been designed to evaluate a patient's personality, along with various other issues. Even though arguments exist on both sides about clinical interview and personal impressions as a psychologist, psychiatrist or a physician, most reliable psychological evaluations are performed by self-report instruments or psychological tests. The most commonly used self-reporting instruments in evaluation of chronic pain are the MMPI, MCMI, Millon Behavioral Health Inventory (MBHI), Symptom Check List-90 (SCL-90), Illness Behavior Questionnaire (IBQ), Beck's Depression Inventory (BDI), Hamilton Rating Scale for Depression, Zung Self-rating Depression Scale, and Modified Somatic Perception Questionnaire (MSPQ), among others. The MCMI-III is considered an appropriate test for personality, as well as diagnosis of clinical syndromes. The MCMI-III is an extensive test providing multiple dimensions of personality, along with issues related to pain management, including all the mood disorders and drug dependence, as well as alcohol dependence, etc. The MCMI-III is a questionnaire with 175 questions compared to the MMPI with 566 questions, and is easily administered, as well as less expensive. Even though MCMI-III could be considered too difficult to perform for routine psychological evaluation and chronic pain patients for screening purposes, this test was utilized for the sole purpose of extensive evaluation in this group of patients compared to normal individuals to identify core differences in personality conditions and clinical syndromes. Some also may question the validity of the MCMI. It is intended to be an evolving assessment to be refined as needed on the basis of substantial advances (41).

Currently, more than 400 research articles have been published that employ the MCMI as a major assessment instrument (41). Numerous cross-validation and cross-generalization studies have been and continue to be executed with the goal of evaluating and improving each of the ele- ments that make up the MCMI: its items, scales, scoring procedures, algorithms, and interpretative text (41). Multiple potential advantages of the MCMI include the relative brevity of the inventory, its theoretical anchoring, its multiaxial format, its construction through three stages of validation, its use of base-rate scores, and its interpretative depth (41). In addition, the findings of multiple investigations into the validity of MCMI interpretative reports provide strong evidence that ratings of its accuracy are higher than can be accounted for by the computer-generated format (41-44). Multiple-item development included not only theory-based structural and functional domains but were also modified to parallel the substantive nature of DSM-IV diagnostic criteria. Internal consistency and test-retest reliability were also demonstrated.

In addition, we may be criticized for utilizing participants in the control group without pain or psychotherapeutic drug therapy and comparing them with pain patients, most of them receiving psychotherapeutic drug therapy. Further, we may be criticized for using the MCMI-III on participants in Group I without psychotherapeutic drug therapy and also without any expected psychiatric disorders. The authors of this study recognize that the MCMI was developed to describe and differentiate among various adult psychiatric patients. It is also recognized that the MCMI was designed to assess personality characteristics and behavioral manifestations that fall outside the normal range of functioning (61). The normative data of the MCMI-III reflect disorder prevalence rates that are unlikely to be found outside mental health settings (61). Millon and Grossman (61) state that the performance of normals on the MCMI-III has yet to be systematically studied. They also reported results of one study designed to investigate the validity of the original MCMI as a screening instrument in a university setting, in which 241 college freshman were administered the MCMI. Using the BR-85 criterion, 75 of these subjects would have been classified as "depressed," greatly exceeding other estimates of prevalence of psychiatric disturbance in an unselected college sample (61). However, we were unable to observe such major abnormalities in the normal population, either with personality patterns, personality pathology, or clinical syndromes. It is also essential to compare nonpain patients without any psychotherapeutic drug therapy to obtain proper data in comparison to pain patients.

We may also face criticism on evaluation of various personality features and severe personality pathology. However, this would be essential to differentiate personality features, personality traits, and personality disorders from severe personality pathology. Personality traits are endur- 
ing patterns of personality, relating to, and thinking of the involvement in oneself that is exhibited in a wide range of social and personal contexts. In contrast, the sensitive future of a personality disorder is an enduring pattern of inexperience and behavior that deviates markedly from the expectations of the individuals and culture and is manifested in at least two of the four areas of cognition, affectivity, interpersonal functioning, or impulse control. This enduring pattern is inflexible and pervasive across a wide, broad range of personal and social situations and leads to clinically significant distress or impairment in social, occupational, or other important areas of functioning. However, the pattern is stable and of long duration, its onset can be traced back at least to adolescence or early adulthood, it is not better accounted as a manifestation or consequence of another medical disorder, and is not due to the direct physiological effects of the substance. In addition, specific diagnostic criteria are also provided for each of the personality disorders.

Finally, a common argument is that depressive symptoms and anxiety symptoms are different from depressive disorders such as major depression, dysthymia, or generalized anxiety disorder. However, in this study, instead of a BR of a certain range, we use the diagnosis as evidenced and provided by the interpretative report.

There are no data available in the medical literature with regards to treatment of personality disorders, somatization disorder, and anxiety disorder in chronic pain in relation to outcomes. However, there is some evidence in the literature showing that depression must and should be treated in the context of general medical conditions, which may improve the prognosis of both depression, as well as the general medical conditions (26). Patients with chronic pain frequently do have serious psychopathology, most often, depressive disorders, anxiety disorders, and somatization disorders, as shown in this study. However, chronic pain should not be discounted or ignored because of its association with psychological disorders. Similarly, psychological disorders should not be discounted or ignored because of this association with chronic pain. In addition, it is also important to recognize that not all patients with chronic pain also suffer with psychological disorders. It is also equally important to recognize that psychological disorders, when present, influence pain and may in turn, be influenced by the level of pain. Thus, it is essential to diagnose psychological disorders prior to embarking on interventional procedures and to try to maximally treat psychological disorders to the best of one's ability and reduce the chronic pain to some extent, specifically if a psycho- logical condition is a confounding factor.

\section{CONCLUSION}

A significant number of patients in the chronic pain group presented with generalized anxiety disorder, somatization disorder, and major depression. In addition, a greater proportion of patients also presented with a psychological disorder in the chronic pain group (55\% vs $24 \%$ ). Hence, we conclude that there may not be a significant relationship between personality patterns and chronic pain, whereas a significant proportion of chronic pain patients do suffer with generalized anxiety disorder, somatization disorder and major depression. Hence, it is recommended that all patients with chronic pain presenting for interventional pain management should undergo at least a screening psychological evaluation to evaluate these three factors, if contraindications do not exist.

\section{REFERENCES}

1. Dersh J, Gatchel RJ, Polatin P. Chronic spinal disorders and psychopathology: Research findings and theoretical considerations. Spine 2001; 1:88-94.

2. Gatchel RJ. A biopsychosocial overview of pretreatment screening of patients with pain. Clin J Pain2001; 17:192-199.

3. Benjamin S, Morris S, McBeth J et al. The association between chronic widespread pain and mental disorder. Arthritis Rheum 2000; 43:561-567.

4. Benjamin S, Barnes D, Berger S et al. The relationship of chronic pain, mental illness and organic disorders. Pain 1988; 32:185-195.

5. Brown GK, Nicassio PM, Wallston KA. Pain coping strategies and depression in rheumatoid arthritis. $J$ Consult Clin Psychol 1989; 57:652-657.

6. Wolfe F. Fibromyalgia. In Sessle BJ, Bryant PS, Dionne RA (eds). Temporomandibular Disorders and Related Pain Conditions. IASP Press, Seattle, 1995, pp 31-46.

7. Bennett RM, Burckhardt CS, Clark SR et al. Group treatment of fibromyalgia. JRheumatol 1996;23:521528.

8. Romano JM, Turner JA. Chronic pain and depression: Does the evidence support a relationship? Psychol Bull 1985; 97:18-34.

9. Barsky AJ, Wyshak G, Klerman GL. Medical and psychiatric determinants of outpatient utilization. Med Care 1986; 24:548-560.

10. Macfarlane GJ, Morris S, Hunt IM et al. Chronic widespread pain in the community: The influence of psychological symptoms and mental disorder on healthcare seeking behaviour. J Rheumatol 1999;26:413419. 
11. Makela M, Heliovaara M. Prevalence of primary fibromyalgia in the Finnish population. BMJ 1991; 303:216-219.

12. Krishnan KRR, France RD, Pelton S et al. Chronic pain and depression. I. Classification of depression in chronic low back pain patients. Pain 1985;22:279287.

13. Von Korff M, Le Resch L, Dworkin S. First onset of common pain symptoms: A prospective study of depression as a risk factor. Pain 1993; 55:251-258.

14. Magni G, Morreschi C, Rigatti-Luchini S et al. Prospective study on the relationship between depressive symptoms and chronic musculoskeletal pain. Pain 1994; 56:289-297.

15. Atkinson JH, Slater MA, Patterson TL et al. Prevalence, onset, and risk of psychiatric disorders in men with chronic low back pain: A controlled study. Pain 1991; 45:111-121.

16. Magni G. Chronic low-back pain and depression: An epidemiologic survey. Acta Psychiatr Scand1984; 70:614-617.

17. Ham LP, Andrasik F, Packard RC et al. Psychopathology in individuals with posttraumatic headaches and other pain types. Cephalgia 1994; 14:118-126.

18. Merikangas KR. Association between psychopathology and headache syndromes. Curr Opin Neurol1995; 8:248-251.

19. Okasha A, Ismail MK, Khalil AH et al. A psychiatric study of non-organic chronic headache patients. Psychomatics 1999; 40:233-238.

20. Kight M, Gatchel RJ, Wesley L. Temporomandibular disorders: Evidence for significant overlap with psychopathology. Health Psychol 1999; 18:177-182.

21. Savidge CJ, Slade P. Psychological aspects of chronic pelvic pain. J Psychosom Res 1997; 42:433-444.

22. Epstein SA, Kay G, Clauw D et al. Psychiatric disorders in patients with fibromyalgia: A multicenter investigation. Psychosomatics 1999; 40:57-63.

23. Polatin PB, Kinney RK, Gatchel RJ et al. Psychiatric illness and chronic low back pain: The mind and the spine-which goes first? Spine 1993;18:66-71.

24. Manchikanti L, Pampati V, Fellows B et al. Characteristics of chronic low back pain in patients in an interventional pain management setting: A prospective evaluation. Pain Physician 2001; 4:131-142.

25. Magni G, Caldieron C, Rigatti-Luchini S et al. Chronic musculoskeletal pain and depressive symptoms in the general population: An analysis of the $1{ }^{\text {st }}$ National Health and Nutrition Examination Survey data. Pain 1990; 43:299-307.

26. Rush AJ, Polatin P, Gatchel RJ. Depression and chronic low back pain. Spine 2000; 25:2566-2571.

27. Ahles TA, Khan SA, Yunus MB et al. Psychiatric status of patients with primary fibromyalgia, patient with rheumatoid arthritis, subjects without pain: A blind comparison of DSM-III diagnoses. Am JPsychiat
1991; 148:1721-1726.

28. Fishbain DA, Cutler R, Rosomoff HL et al. Chronic pain associated depression: Antecedent or consequence of chronic pain? A review. Clin J Pain 1997; 13:116137.

29. Main CJ, Waddell G. Psychiatric distress. In Waddell G (ed). The Back Pain Revolution. Churchill Livingstone, Philadelphia, 1998, pp 173-186.

30. Sullivan M, Katon W. Somatization: the path between distress and somatic symptoms. Am Pain Soc J1993; 2:141-149.

31. Barsky AJ, Klerman GL. Overview: hypochondriasis, bodily complaints, and somatic styles. Am J Psychiat 1983; 140:273-283.

32. Davis PJ, Reeves JL, Hastie BA et al. Depression determines illness conviction and pain impact: A structural equation modeling analysis. Pain Med2000; $1: 238-246$.

33. Epker J, Block AR. Presurgical psychological screening in back pain patients: A review. Clin J Pain 2001; 17:200-205.

34. Carragee EJ. Psychological screening in the surgical treatment of lumbar disc herniation. Clin J Pain 2001; 17:215-219.

35. Wells KB, Sherbourne C, Shoenbaum Met al. Impact of disseminating quality improvement programs for depression in managed primary care. A randomized controlled trial. JAMA 2000;283;212-220.

36. Depression Guidelines Panel. Depression in Primary Care, I: Detection and Diagnosis. Rockville, MD. US Dept of Health and Human Services, 1993. US Public Health Service, Agency for Health Care Policy and Research publication 93-0550.

37. Depression Guidelines Panel. Depression in Primary Care, II: Treatment of Major Depression. Rockville, MD, US Dept of Health and Human Services; 1993. US Public Health Service, Agency for Health Care Policy and Research publication 93-0551.

38. Schulberg HC, Block MR, Madonia MJ et al. Treating major depression in primary care practice: 8-month clinical outcomes. Arch Gen Psychiatry 1996; 53:913919.

39. Malt UF, Robak OH, Madsbu H-P et al. The Norwegian Naturalistic Treatment Study of Depression in General Practice (NORDEP)-I: Randomized double blind study. BMJ 1999;318:1180-1184.

40. Attikson CC, Zich JM. Depression in Primary Care: Screening and Detection. New York, NY, Routledge, 1990.

41. Millon T. MCMI-III Manual, ed 2, NCS, Inc., Minneapolis, 1997.

42. Craig RJ (ed). The Millon Clinical Multiaxial Inventory: A Clinical Research Information Synthesis. Lawrence Erlbaum, Hillsdale.

43. Moreland KL, Onstad JA. Validity of Millon's computerized interpretation system for the MCMI: A con- 
trolled study. J Consulting Clin Psychology 1987; 55:113-114.

44. Sandberg ML. Is the ostensive accuracy of computer interpretive reports a result of the Barnum effect? A study of the MCMI. In C. Green (ed). Conference on the Millon Clinical Inventories (MCMI, MBHI, MAPI), Minneapolis National Computer Systems, 1987, pp 155-164.

45. Kramlinger KG, Swanson DW, Maruta T. Are patients with chronic pain depressed? Am J Psychiatry 1983; 140:747-749.

46. Weisberg JN, Keefe FJ. Personality disorders in the chronic pain population: Basic concepts, empirical findings, and clinical implications. Pain Forum 1997; 6:1-9.

47. Engel GL. "Psychogenic" pain and the pain-prone patient. Am J Med 1959; 26:899-918.

48. Hanvik LJ. MMPI profiles in patients with low back pain. J Consult Psychol 1951;15:350-353.

49. Sternbach RA, Wolf SR, Murphy RW et al. Aspects of chronic low back pain. Psychosomatics 1973; 14:5256.

50. Szasz TS. Pain and pleasure: A Study of Bodily Feelings. Tavistock, 1957.

51. Woodforde JM, Merskey H. Personality traits of patients with chronic pain. J Psychosom Res 1972; 16:167-172.

52. Narrow WE, Rae DS, Regier DA. NIMH epidemiology note: Prevalence of Anxiety Disorders. One-year Prevalence Best Estimates Calculated from ECA and NCS data. Population Estimates Based on U.S. Census Estimated Residential Population Age 18 to 54 on July 1, 1998.

53. Asmundson GJ, Jacobson SJ, Allerdings MD et al. Social phobia in disabled workers with chronic musculoskeletal pain. Behav Res Ther 1996; 34:939-943.

54. Regier DA, Narrow WE, Rae DS et al. The de facto mental and addictive disorders service system. Epidemiologic Catchment Area prospective 1-year prevalence rates of disorders and services. Arch Gen Psychiatry 1993; 50:85-94.

55. Narrow WE. One-year prevalence of mental disorders, excluding substance use disorders, in the U.S.: NIMH ECA prospective data. Population estimates based on U.S. Census estimated residential population age 18 and over on July 1, 1998.

56. Murray CJ, Lopez AD (eds). The Global Burden of Disease: A Comprehensive Assessment of Mortality and Disability from Diseases, Injuries, and Risk Factors in 1990 and Projected to 2020. Cambridge, MA; Harvard School of Public Health on Behalf of the World Health Organization and the World Bank, Harvard University Press, 1996. http://www.who.int/ msa/mnh/ems/dalys/intro.htm

57. Aronoff GM. Psychodynamics and psychotherapy of the chronic pain syndrome. In Aronoff GM (ed). Evaluation and Treatment of Chronic Pain, ed 3. Williams and Wilkins, Philadelphia, 1999, pp 83290.

58. Fink P, Sorensen L, Engberg M et al. Somatization in primary care. Prevalence, health care utilization, and general practitioner recognition. Psychosomatics 1999; 40:330-338.

59. Katon W, Egan K, Millder D. Chronic pain: Lifetime psychiatric diagnosis and family history. Am JPsychiatry $1985 ; 142: 1156-1160$.

60. Waddell G, Turk DC. Clinical assessment of low back pain. In Turk DC, Melzack R (eds). Handbook of Pain Assessment. First Edition. The Guilford Press, New York, 1992, pp 15-36.

61. Millon T, Grossman SD. Conducting Publishable $M C M C-I I I \circledR$ research. National Computer Systems, Minneapolis, 2000. 